\title{
Modeling Pauli measurements on graph states with nearest-neighbor classical communication
}

\author{
Jonathan Barrett, ${ }^{1, *}$ Carlton M. Caves, ${ }^{2}$ Bryan Eastin, ${ }^{2}$ Matthew B. Elliott, ${ }^{2, \dagger}$ and Stefano Pironio ${ }^{3, *}$ \\ ${ }^{1}$ Perimeter Institute for Theoretical Physics, 31 Caroline Street N, Waterloo, Ontario, Canada N2L $2 Y 5$ \\ ${ }^{2}$ Department of Physics and Astronomy, University of New Mexico, Albuquerque, New Mexico 87131, USA \\ ${ }^{3}$ ICFO-Institut de Ciències Fotòniques, Mediterranean Technology Park, 08860 Castelldefels (Barcelona), Spain \\ and Institute for Quantum Information, California Institute of Technology, Pasadena, California 91125, USA
}

(Received 6 March 2006; revised manuscript received 5 September 2006; published 8 January 2007)

\begin{abstract}
We propose a communication-assisted local-hidden-variable model that yields the correct outcome for the measurement of any product of Pauli operators on an arbitrary graph state, i.e., that yields the correct global correlation among the individual measurements in the Pauli product. Within this model, communication is restricted to a single round of message passing between adjacent nodes of the graph. We show that any model sharing some general properties with our own is incapable, for at least some graph states, of reproducing the expected correlations among all subsets of the individual measurements. The ability to reproduce all such correlations is found to depend on both the communication distance and the symmetries of the communication protocol.
\end{abstract}

DOI: 10.1103/PhysRevA.75.012103

PACS number(s): 03.65.Ud, 03.67.-a

\section{INTRODUCTION}

Graph states are multipartite entangled states that play many important roles in quantum information theory. The class of graph states is equivalent, by local unitaries in the Clifford group, to the class of states stabilized by Pauli operators $[1,2]$. This class includes Bell states, GHZ states, basis states for stabilizer codes, cluster states, and many others. Of particular interest are the cluster states, which are the graph states represented by two-dimensional square lattices [3]. Cluster states have been shown to be sufficient to allow universal quantum computation within a measurement-based scheme [4]. For this reason, a complete understanding of the entanglement properties of graph states would likely improve our understanding of the role entanglement plays in quantum computation, as well as teaching us about some of the most useful states in quantum information theory. Graph states and their applications are reviewed in Ref. [5].

Both Gühne et al. [6] and Scarani et al. [7] have shown that graph states display nonlocal properties under the measurement of Pauli operators. In this work, we further our understanding of the nonlocality of graph states by introducing a communication-assisted local-hidden-variable (LHV) model that predicts the outcome of measuring an arbitrary Pauli product on an arbitrary graph state. Since graph states violate Bell-type inequalities, the model necessarily involves communication.

Our investigation is inspired by that of Tessier et al. [8], who described a communication-assisted LHV model for arbitrary Pauli measurements on a GHZ state. In the spirit of Tessier et al., we formulate our LHV model in terms of hidden variables that can be thought of as specifying values for the $x, y$, and $z$ spin components of the qubits. In general, a communication protocol might permit the party at a particu-

\footnotetext{
*Electronic address: jbarrett@perimeterinstitute.ca

†Electronic address: mabellio@unm.edu

"Electronic address: Stefano.Pironio@icfo.es
}

lar qubit to communicate to any other party what Pauli measurement is made on its qubit. In our communication protocol, however, we restrict communication to be between parties corresponding to nodes that are adjacent in the underlying graph. This restriction to communication only with neighbors in the graph makes intuitive sense if we think of a graph as a recipe for constructing the corresponding graph state. In that case, nodes that are connected have interacted in the past and therefore occupy a privileged position with regard to exchange of information. We call a protocol that restricts communication to neighbors a nearest-neighbor communication protocol.

Although our communication-assisted LHV model predicts correctly the outcome of the measurement of any Pauli product, it fails in some cases to predict the expected correlations for subsets of the individual measurements in a Pauli product. By considering restricted classes of graphs, we show that two general properties of our model assure its failure. Perhaps unsurprisingly, one of these is the limitation to nearest-neighbor communication. More generally, we consider protocols with a limited communication distance, defined as the number of successive edges through which information can be sent, and we show that any protocol whose communication distance is constant or scales less than linearly with the number of qubits fails to predict some submeasurements correctly. Less obvious is a second problem of our protocol, which we call site invariance, i.e., the property that nodes in symmetric situations perform the same action. We consider the effects of each of these properties in some detail and show that if a protocol has either property, it fails on some submeasurements.

This paper is organized as follows. In Sec. II we introduce the formal definition of graph states. In Sec. III we describe our model and prove that it correctly predicts the global result of any Pauli measurement on a graph state, i.e., predicts the global correlation among the individual measurements in the Pauli product. In Sec. IV we demonstrate that neither site invariance nor any fixed communication distance is compatible with the goal of reproducing all subcorrelations, though we do demonstrate that a site-invariant protocol can repro- 
duce all subcorrelations on a one-dimensional cluster state. A final section summarizes our conclusions.

\section{GRAPH STATES}

A graph is a set of $n$ nodes and a set of edges connecting them. The neighborhood $\mathcal{N}(j)$ of a node $j$ is the set of nodes that are connected to it. Given a particular graph, we can associate a qubit with each node and define the corresponding graph state of the qubits in the following way. Let $X, Y$, and $Z$ denote the Pauli matrices $\sigma_{x}, \sigma_{y}$, and $\sigma_{z}$, and adopt the shorthand of writing tensor products of Pauli matrices as products of Pauli matrices indexed by position, i.e., $X \otimes I \otimes Y=X_{1} Y_{3}$. The graph state $|\psi\rangle$ on $n$ qubits is the simultaneous +1 eigenstate of the (commuting) operators

$$
G_{j}=X_{j} \prod_{k \in \mathcal{N}(j)} Z_{k}, \quad j=1, \ldots, n .
$$

The operators $G_{j}$ constitute an independent set of generators of the stabilizer group of $|\psi\rangle$. Any graph state can be constructed by preparing each qubit in the eigenstate of spin up in the $x$ direction and then applying a controlled-phase gate between each pair of qubits that is connected by an edge in the graph. The order in which the controlled-phase gates are implemented is unimportant since they all commute.

The structure of graph states makes them good candidates for the study of nonlocality. For a connected graph (of at least two nodes), all single-qubit measurements yield random values, yet these values are correlated in such a way that certain products of them give deterministic results. If $M$ represents an $n$-fold tensor product of the Pauli matrices, $I, X, Y$, and $Z$, then the result of measuring $M$ on the $n$-qubit graph state $|\psi\rangle$ is determined by which of following three cases applies to $M$ (see Ref. [9]).

(i) $M$ is an element of the stabilizer group, i.e., $M=G_{1}^{a_{1}} \cdots G_{n}^{a_{n}}$ is a product of the generators $G_{j}$ for some $a_{j}=0,1$, in which case a measurement of $M$ obviously gives outcome +1 .

(ii) $-M$ is an element of the stabilizer group, i.e., $-M=G_{1}^{a_{1}} \cdots G_{n}^{a_{n}}$ is a product of the generators $G_{j}$ for some $a_{j}=0,1$, in which case a measurement of $M$ obviously gives outcome -1 .

(iii) $\pm M$ is not an element of the stabilizer, i.e., $M$ is not a product of the generators up to a multiplicative factor \pm 1 , in which case a measurement of $M$ gives outcomes +1 and -1 with equal probability.

The minus sign in case (ii) comes from the fact that products of generators can introduce at each site terms such as $Z X Z=-X$ or $Z X=i Y$, with $i$ 's from pairs of sites multiplying to give a -1 . These terms lead to GHZ-like paradoxes for the graph state, implying that communication between the parties is required to model the correlations classically.

\section{COMMUNICATION-ASSISTED LHV MODEL FOR GRAPH STATES}

\section{A. Description of the model}

Our model uses $n$ binary random variables, $z_{1}, \ldots, z_{n}$, each taking on values \pm 1 with equal probability. These hid- den variables can be thought of as values for the $z$ spin components of the $n$ qubits. For the corresponding values of the $x$ and $y$ spin components, we define the quantities

$$
\begin{gathered}
x_{j}=\prod_{k \in \mathcal{N}(j)} z_{k}, \\
y_{j}=z_{j} \prod_{k \in \mathcal{N}(j)} z_{k} .
\end{gathered}
$$

The values $x_{j}$ are suggested by the +1 values associated with the generators $G_{j}$, i.e.,

$$
x_{j} \prod_{k \in \mathcal{N}(j)} z_{k}=+1,
$$

in analogy to Eq. (1). The values

$$
y_{j}=x_{j} z_{j}
$$

are suggested by the analogous relations $Y_{j}=i X_{j} Z_{j}$ for Pauli matrices.

We assume now that each party is given a measurement $M_{j}$ to perform, chosen from $I$ (no measurement), $X, Y$, and $Z$. After the measurement, there is a round of communication between neighboring sites, and then each party outputs a value +1 or -1 as the result of the measurement. When no measurement is performed at a site, the output can be regarded as +1 .

During the round of communication, site $j$ sends a bit $c_{j}$ to each site $k \in \mathcal{N}(j)$, where $c_{j}=0$ if $M_{j}=I, Z$ and $c_{j}=1$ if $M_{j}=X, Y$. The value $v_{j}$ output at site $j$ is determined by the hidden variable for the observable measured at that site and by the quantity

$$
t_{j}=\sum_{k \in \mathcal{N}(j)} c_{k} \bmod 4
$$

which is computed from the bits sent to site $j$ from neighboring sites and which is equal to the number of neighboring sites that make an $X$ or $Y$ measurement modulo 4. The output $v_{j}$ is determined by rules that decide whether to flip the sign of the hidden variable associated with the measurement at site $j$.

(1) If $M_{j}=I, v_{j}=1$.

(2) If $M_{j}=Z, v_{j}=z_{j}$.

(3) If $M_{j}=X, v_{j}= \begin{cases}x_{j} & \text { if } t_{j}=0,1, \\ -x_{j} & \text { if } t_{j}=2,3 \text {. }\end{cases}$

(4) If $M_{j}=Y, v_{j}= \begin{cases}y_{j} & \text { if } t_{j}=1,2, \\ -y_{j} & \text { if } t_{j}=0,3 \text {. }\end{cases}$

This protocol reproduces the quantum predictions for any global Pauli measurement on graph states, as we show in the next subsection. In other words, if we take the product of the outputs from all the sites, the result is the same as the quantum prediction for a measurement of the operator $M=\otimes_{j=1}^{n} M_{j}$. The number of bits communicated in this protocol is twice the number of edges in the graph.

Variants of rules (3) and (4) also give the correct predictions for global Pauli measurements; for example, these rules can be modified so that the sign flip occurs under the same 
circumstances for both $X$ and $Y$ measurements. We note, however, that neither the rules given above nor these modified rules are guaranteed to reproduce all of the correlations predicted by quantum mechanics on subsets of the Pauli operators measured. We take up the question of these subcorrelations in Sec. IV.

\section{B. Proof that the model works}

The proof that our model yields the correct global quantum predictions proceeds in two stages. We first introduce a simple related model that involves no classical communication and show that this simple model makes the correct quantum predictions in cases (i) and (iii) above, but not in case (ii). We then show that the communication-assisted model makes correct global predictions in all three cases.

The simple no-communication model has each party output the hidden variable $1, x_{j}, y_{j}$, or $z_{j}$ associated with the measurement made at its site. The communication-assisted model is derived from this no-communication model by sometimes flipping the sign of the outcome at a site where $X$ or $Y$ is measured, i.e., by outputting $-x_{j}$ instead of $x_{j}$ or $-y_{j}$ instead of $y_{j}$. The decision to flip a sign is determined by the number of $X$ and $Y$ measurements made at neighboring sites, in accordance with the conditions in rules (3) and (4).

For any tensor product of Pauli operators, $M=\otimes_{j=1}^{n} M_{j}$, it is useful to introduce a corresponding $n$-tuple $m$ of the same form as $M$, but with the tensor product of Pauli operators replaced by an $n$-tuple of the corresponding hidden variables, $1, x_{j}, y_{j}$, and $z_{j}$. In the no-communication model, the elements of the $n$-tuple are the outcomes of the measurements $M_{j}$. The $n$-tuples $m$ form an Abelian group of order $4^{n}$, with multiplication defined bitwise.

The hidden variables $x_{j}, y_{j}, z_{j}$ satisfy a commutative algebra, similar to the Pauli algebra, with $x_{j}^{2}=y_{j}^{2}=z_{j}^{2}=1$ and $y_{j}=x_{j} z_{j}$, as in Eq. (4). The noteworthy differences from the Pauli algebra are the commutativity and the absence of an $i$ in Eq. (4). As a consequence, when a measurement has the form $M= \pm G_{1}^{a_{1}} \cdots G_{n}^{a_{n}}$, the product of all parties' outputs in the no-communication model always equals +1 . Thus it is clear that the no-communication model gets the correct result in case (i) above, but not in case (ii).

We show now that the no-communication model is also correct in case (iii). For this purpose, note that the $n$-tuples $g_{j}$ associated with the stabilizer generators $G_{j}$ generate a subgroup of order $2^{n}$, which contains the $n$-tuples associated with all Pauli products $M$ such that $\pm M$ is in the stabilizer. This subgroup defines $2^{n}$ cosets which, except for the subgroup itself, necessarily contain $n$-tuples associated with Pauli products from case (iii). Thus we need to show that the no-communication model predicts a random outcome for all cosets except the subgroup itself. We note that two $n$-tuples in the same coset predict the same outcome, thus allowing us to restrict attention to a single element in each coset. Elements of the form $\left(z_{1}^{a_{1}}, \ldots, z_{n}^{a_{n}}\right)$, with $a_{j}=0,1$, clearly predict a random overall outcome, except when $a_{j}=0$ for all $j$ (i.e., the identity $n$-tuple). Moreover, these $2^{n} n$-tuples each belong to a different coset, since they make up a subgroup of their own that contains none of the elements of the subgroup gen- erated by the $g_{j}$, except the identity. Thus we recover the correct predictions for case (iii).

The next step in the proof is to show that the communication-assisted LHV model recovers the correct predictions for a measurement of $M$ in all three cases. If $M$ is as in (iii), then the result predicted by the no-communication model is random, and flipping an outcome at any site does not affect this. Thus the communication model works when $M$ is as in (iii). To show that the model also works when $M$ is as in (i) or (ii), we proceed by induction. The model works when $M$ is any one of the generators $G_{j}$. We consider a Pauli product $M=\otimes_{l=1}^{n} M_{l}$ that is a product, up to a factor \pm 1 , of generators $G_{k}$ with $k<j$. With this assumption, it is clear that $M_{j}$ is either $I$ or $Z$. Our inductive procedure is to show that if the model correctly predicts the overall correlation for $M$, then it also reproduces the overall correlation for $M^{\prime}= \pm M G_{j}$.

Consider the outcome for a measurement of $M^{\prime}$, as predicted by quantum mechanics. We express $M^{\prime}$ in terms of $M=\otimes_{l=1}^{n} M_{l}$ and the generator $G_{j}=X_{j} \Pi_{k \in \mathcal{N}(j)} Z_{k}$. Upon multiplying $M$ with $G_{j}$, we obtain the following: (a) At each $k \in \mathcal{N}(j)$ for which $M_{k}=I$, the product $I Z=Z$ gives $M_{k}^{\prime}=Z$; (b) at each $k \in \mathcal{N}(j)$ for which $M_{k}=Z$, the product $Z Z=I$ gives $M_{k}^{\prime}=I$; (c) at each $k \in \mathcal{N}(j)$ for which $M_{k}=X$-we let $q$ denote the number of such sites-the product $X Z=-i Y$ gives $M_{k}^{\prime}=Y$ and introduces a factor of $-i$; (d) at each $k \in \mathcal{N}(j)$ for which $M_{k}=Y$-we let $r$ denote the number of such sites-the product $Y Z=i X$ gives $M_{k}^{\prime}=X$ and introduces a factor of $+i$. Overall we thus obtain a factor $(-i)^{q} i^{r}=(-i)^{q+r}(-1)^{r}$. Now consider site $j$ : If $q+r$ is even, $M_{j}=I$, and we are left with $M_{j}^{\prime}=X$ and no additional factors of $i$; if $q+r$ is odd, $M_{j}=Z$, and we are left with $M_{j}^{\prime}=Y$ and an additional factor of $i$. There are thus four possibilities: If $q+r=0,1 \bmod 4$, then $M^{\prime}=(-1)^{r} M G_{j}$, and if $q+r=2,3 \bmod 4$, then $M^{\prime}$ $=(-1)^{r+1} M G_{j}$. It follows that in the case $q+r=0 \bmod 4$, the quantum prediction for measurement of the operator $M^{\prime}$ is equal to the quantum prediction for a measurement of $M$ multiplied by $(-1)^{r}$, and similarly for the other cases.

Now we consider the prediction given for a measurement of $M^{\prime}$ by our communication-assisted LHV model, assuming that the correct prediction is returned for $M$. The value returned for a measurement of $M^{\prime}$ is equal to the value returned for a measurement of $M$, multiplied by the value returned for a measurement of $G_{j}$, which is 1 , and by a -1 for each site that changes its sign-flip decision. A review of the immediately preceding paragraph shows that the only site that changes the $c$-bit sent to neighboring sites is site $j$, which changes its $c$-bit from $c_{j}=0$ to $c_{j}=1$. This means that at neighboring sites $k \in \mathcal{N}(j)$, the quantity $t_{k}$ of Eq. (5) increases by 1 . At neighboring sites $k \in \mathcal{N}(j)$ for which $M_{k}$ $=X$, the $X$ becomes a $Y$ in $M^{\prime}$, with $t_{k}$ increased by 1 , so according to rules (3) and (4), there is no change in the sign-flip decision. At neighboring sites $k \in \mathcal{N}(j)$ for which $M_{k}=Y$, the $Y$ becomes a $X$ in $M^{\prime}$, with $t_{k}$ increased by 1 , so according to rules (3) and (4), site $k$ changes its sign-flip decision. The result of these changes is an overall factor of $(-1)^{r}$. The final contribution comes from site $j$, for which $t_{j}=q+r \bmod 4$, and which changes from $M_{j}=I$ to $M_{j}^{\prime}=X$ if $q+r$ is even, and from $M_{j}=Z$ to $M_{j}^{\prime}=Y$ if $q+r$ is odd. Ac- 
cording to the rules, the effect of these changes is to introduce an additional sign flip if and only if $q+r=2,3 \bmod 4$, which is just what is required to return the quantum predictions.

\section{GRAPH-STATE SUBMEASUREMENTS}

Having shown that our communication-assisted LHV model agrees with quantum mechanics for global correlations, we now consider the question of submeasurements. A submeasurement of a global Pauli product $M$ is a Pauli product $\tilde{M}$ such that the nonidentity elements of $\tilde{M}$ all appear in $M$, i.e., $\tilde{M}_{j}=M_{j}$ or $\tilde{M}_{j}=I$ for all $j$. LHV models implicitly predict the result of measuring such a subset of the Pauli operators of a global measurement, the measurement of an identity operator being simply the omission of the corresponding local hidden variable. A proper communicationassisted LHV model for graph states should not only reproduce the predictions of quantum mechanics for global measurements but also for all possible submeasurements.

It can be shown that our model satisfies this condition for some, but not all, graph states. Determining the graphs for which it works, a class including complete bipartite graphs (a case encompassing the star graphs of GHZ states) and the symmetric difference of two complete graphs [10], requires the introduction of techniques otherwise unused in this paper, and, as such, we reserve its exposition for another time [11]. Instead, we focus here on understanding the properties that limit our model's effectiveness. The following two subsections show that protocols with fixed communication distance or with symmetric communication and decision protocols generally do not reproduce all subcorrelations on all graphs. The final subsection further explores the symmetry of site invariance by considering it in the context of onedimensional cluster states.

\section{A. Non-nearest-neighbor communication protocols}

In a communication protocol with communication distance $d$, nodes $j$ and $k$ can signal to each other if there exists within the graph a path from $j$ to $k$ that traverses $d$ or fewer edges [10]. Put another way, this is the statement that information can only be transmitted along edges and that the number of successive edges through which some piece of information can be sent is at most $d$. In this section we prove, via contradiction, that no communication-assisted LHV model for which the communication distance satisfies

$$
d \leqslant 4\left\lfloor\frac{n}{24}-\frac{1}{2}\right\rfloor+1
$$

correctly reproduces the predictions of quantum mechanics for all submeasurements on all graph states of $n$ qubits.

The proof relies on an infinite class of graph states for which a set of five global measurements can be chosen that are not locally distinguishable. Each of these global measurements includes a submeasurement that can be written in terms of stabilizer elements and is thus certain. The output of each qubit, however, must be such that the correct values are obtained for all submeasurements that are consistent with its

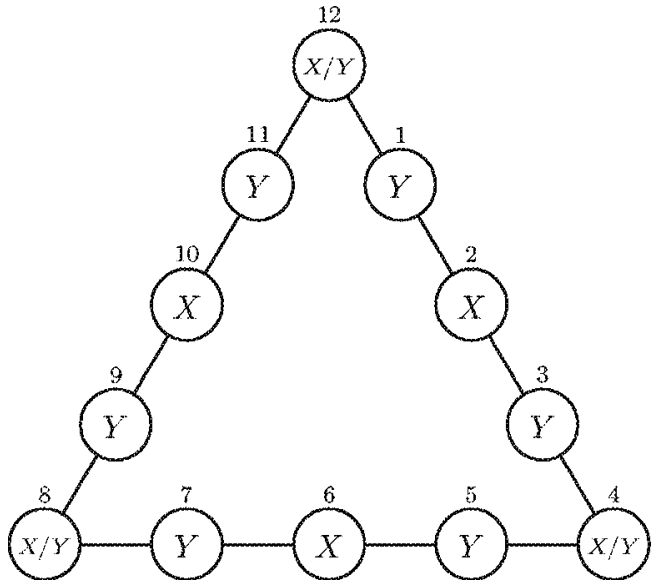

FIG. 1. Example demonstrating that any communicationassisted LHV model with communication distance $d \leqslant 1$ fails to reproduce some submeasurements for the ring with $n=12$ nodes. Five of the global measurements shown on the ring,

$$
\begin{aligned}
& Y_{1} \underline{X}_{2} Y_{3} \underline{X}_{4} Y_{5} \underline{X}_{6} Y_{7} \underline{X}_{8} Y_{9} \underline{X}_{10} Y_{11} \underline{X}_{12}, \\
& \underline{Y}_{1} \underline{X}_{2} \underline{Y}_{3} Y_{4} \underline{Y}_{5} \underline{X}_{6} \underline{Y}_{7} Y_{8} \underline{Y}_{9} \underline{X}_{10} \underline{Y}_{11} Y_{12}, \\
& \underline{Y}_{1} X_{2} \underline{Y}_{3} \underline{Y}_{4} Y_{5} \underline{X}_{6} Y_{7} \underline{X}_{8} Y_{9} \underline{X}_{10} Y_{11} \underline{Y}_{12}, \\
& Y_{1} \underline{X}_{2} Y_{3} \underline{Y}_{4} \underline{Y}_{5} X_{6} \underline{Y}_{7} \underline{Y}_{8} Y_{9} \underline{X}_{10} Y_{11} \underline{X}_{12}, \\
& Y_{1} \underline{X}_{2} Y_{3} \underline{X}_{4} \underline{Y}_{5} \underline{X}_{6} \underline{Y}_{7} \underline{Y}_{8} \underline{Y}_{9} \bar{X}_{10} \underline{Y}_{11} \underline{Y}_{12},
\end{aligned}
$$

contain submeasurements (underlined) useful for showing a contradiction. These submeasurements imply the following constraints on a nearest-neighbor communication model, $x_{2} x_{4} x_{6} x_{8} x_{10} x_{12}=1, y_{1}^{Y} x_{2} y_{3}^{Y} y_{5}^{Y} x_{6} y_{7}^{Y} y_{9}^{Y} x_{10} y_{11}^{Y}=-1, y_{1}^{Y} y_{3}^{Y} y_{4} x_{6} x_{8} x_{10} y_{12}=1$, $x_{2} y_{4} y_{5}^{Y} y_{7}^{Y} y_{8} x_{10} x_{12}=1, x_{2} x_{4} x_{6} y_{8} y_{9}^{Y} y_{11}^{Y} y_{12}=1$, which, when multiplied together, yield a contradiction.

observable surroundings. This requirement, for the particular states and measurements chosen, yields a contradiction.

To begin, consider the graph state corresponding to an $n$-node ring where $n=12 f$ and $f$ is an odd positive integer. Let the qubits be numbered sequentially, starting with 1 at an arbitrary point on the ring and moving clockwise along it. Additionally, define the following subsets of the $n$ labels:

$$
\begin{gathered}
\mathcal{V}=\{4 f, 8 f, 12 f\}, \\
\mathcal{M}=\{2 f, 6 f, 10 f\}, \\
\mathcal{Y}=\{j \mid j \equiv 1 \bmod 2\}, \\
\mathcal{L}=\{j \mid j \notin \mathcal{V}, \mathcal{M} \quad \text { and } j \equiv 2 \bmod 4\}, \\
\mathcal{R}=\{j \mid j \notin \mathcal{V}, \mathcal{M} \text { and } j \equiv 0 \bmod 4\}, \\
\mathcal{S}_{k}=\{j \mid 2 f(k-1)<j<2 f k\} .
\end{gathered}
$$

For our purposes, it is useful to think of the ring as arranged in an equilateral triangle with vertices specified by the subset $\mathcal{V}$ (see Fig. 1). The midpoints of the legs of the triangle are then given by the subset $\mathcal{M}$, and the segments between adjacent vertices and midpoints are given by the $\mathcal{S}_{j}$ 's. We use the notation $\mathcal{S}_{j, k}$ as shorthand for $\mathcal{S}_{j} \cup \mathcal{S}_{k}$, and $\mathcal{A} \backslash \mathcal{B}$ is used to denote the set consisting of the elements of $\mathcal{A}$ that are not in $\mathcal{B}$. 
Now consider global measurements of the form

$$
M_{j}= \begin{cases}X \text { or } Y & \text { if } j \in \mathcal{V}, \\ Y & \text { if } j \in \mathcal{Y}, \\ X & \text { otherwise, }\end{cases}
$$

such that the number of vertices measuring $Y$ is not one. These global measurements include the following submeasurements, for which quantum mechanics predicts an outcome with certainty.

$$
\begin{aligned}
& \text { For } M_{4 f}=X, M_{8 f}=X, M_{12 f}=X, \\
& \qquad X_{2 f} X_{4 f} X_{6 f} X_{8 f} X_{10 f} X_{12 f} \prod_{j \in \mathcal{L} \cup \mathcal{R}} X_{j}=\prod_{j=1}^{6 f} G_{2 j},
\end{aligned}
$$

implying a measurement outcome of +1 .

For $M_{4 f}=Y, M_{8 f}=Y, M_{12 f}=Y$,

$$
X_{2 f} X_{6 f} X_{10 f} \prod_{j \in \mathcal{Y}} Y_{j} \prod_{k \in \mathcal{L}} X_{k}=\prod_{j=1}^{3 f}\left(-G_{4 j-3} G_{4 j-2} G_{4 j-1}\right),
$$

implying a measurement outcome of $(-1)^{3 f}=-1$.

For $M_{4 f}=Y, M_{8 f}=X, M_{12 f}=Y$,

$$
\begin{array}{r}
Y_{4 f} X_{6 f} X_{8 f} X_{10 f} Y_{12 f} \prod_{j \in \mathcal{Y} \cap \mathcal{S}_{1,2}} Y_{j} \prod_{k \in \mathcal{R} \cup\left(\mathcal{L} \backslash \mathcal{S}_{1,2}\right)} X_{k} \\
=G_{1} \prod_{j=1}^{f-1}\left(-G_{4 j-1} G_{4 j} G_{4 j+1}\right) G_{4 f-1} \prod_{k=2 f}^{6 f} G_{2 k},
\end{array}
$$

implying a measurement outcome of $(-1)^{f-1}=+1$.

Cyclic permutation of this last measurement yields two more with +1 outcomes.

For $M_{4 f}=Y, M_{8 f}=Y, M_{12 f}=X$, we have

$$
X_{2 f} Y_{4 f} Y_{8 f} X_{10 f} X_{12 f} \prod_{j \in \mathcal{Y} \cap \mathcal{S}_{3,4}} Y_{j} \prod_{k \in \mathcal{R} \cup\left(\mathcal{L} \backslash \mathcal{S}_{3,4}\right)} X_{k},
$$

and for $M_{4 f}=X, M_{8 f}=Y, M_{12 f}=Y$, we have

$$
X_{2 f} X_{4 f} X_{6 f} Y_{8 f} Y_{12 f} \prod_{j \in \mathcal{Y} \cap \mathcal{S}_{5,6}} Y_{j} \prod_{k \in \mathcal{R} \cup\left(\mathcal{L} \backslash \mathcal{S}_{5,6}\right)} X_{k} .
$$

Now assume there exists a distance $d=2 f-1$ communication-assisted LHV model that correctly replicates the predictions of quantum mechanics for all Pauli measurements on $n$ qubits. The output of such a model can be fully described in terms of single-qubit hidden variables whose value depends both on the qubit in question and on the measurements made by other qubits within its communication range. We write these hidden variables in the form $\sigma_{j}{ }^{\alpha}$ where $j$ is the qubit being measured, $\sigma$ is the hidden variable corresponding to the Pauli operator measured upon it, and $\alpha$ indicates the measurements made on qubits within its communication range. The global measurements utilized for Eqs. (9) have the virtue that each qubit's communication range includes at most one other qubit whose measurement is changeable, and that is the qubit at the nearest vertex. Thus, in comparisons between them, the measurement performed on, at most, a single qubit need be included in $\alpha$. Moreover, the qubits at the center of each side of the triangle cannot see the changes at the vertices. Consequently, the constraints im- plied by Eqs. (9) on a hidden variable model with communication range $d$ can be expressed as follows:

$$
\begin{aligned}
1=x_{2 f} x_{4 f} x_{6 f} x_{8 f} x_{10 f} x_{12 f} \prod_{j \in \mathcal{L} \cup \mathcal{R}} x_{j}^{X}, \\
-1=x_{2 f} x_{6 f} x_{10 f} \prod_{j \in \mathcal{Y}} y_{j}^{Y} \prod_{k \in \mathcal{L}} x_{k}^{Y}, \\
1=y_{4 f} x_{6 f} x_{8 f} x_{10 f} y_{12 f} \\
\quad \times \prod_{j \in \mathcal{Y} \cap \mathcal{S}_{1,2}} y_{j}^{Y} \prod_{k \in(\mathcal{L} \cup \mathcal{R}) \cap \mathcal{S}_{4,5}} x_{k}^{X} \prod_{l \in\left(\mathcal{L} \cap \mathcal{S}_{3,6}\right) \cup\left(\mathcal{R} \backslash \mathcal{S}_{4,5}\right)} x_{l}^{Y}, \\
1=x_{2 f} y_{4 f} y_{8 f} x_{10 f} x_{12 f} \\
\quad \times \prod_{j \in \mathcal{Y} \cap \mathcal{S}_{3,4}} y_{j}^{Y} \prod_{k \in(\mathcal{L} \cup \mathcal{R}) \cap \mathcal{S}_{6,1}} x_{k}^{X} \prod_{l \in\left(\mathcal{L} \cap \mathcal{S}_{5,2}\right) \cup\left(\mathcal{R} \backslash \mathcal{S}_{6,1}\right)} x_{l}^{Y}, \\
1= \\
\quad x_{2 f} x_{4 f} x_{6 f} y_{8 f} y_{12 f} \\
\quad \times \prod_{j \in \mathcal{Y} \cap \mathcal{S}_{5,6}} y_{j}^{Y} \prod_{k \in(\mathcal{L} \cup \mathcal{R}) \cap \mathcal{S}_{2,3}} x_{k}^{X} \prod_{l \in\left(\mathcal{L} \cap \mathcal{S}_{1,4}\right) \cup\left(\mathcal{R} \backslash \mathcal{S}_{2,3}\right)} x_{l}^{Y} .
\end{aligned}
$$

Using the identity $\mathcal{A}=\mathcal{A} \cap\left(\mathcal{S}_{1} \cup \mathcal{S}_{2} \cup \mathcal{S}_{3} \cup \mathcal{S}_{4} \cup \mathcal{S}_{5} \cup \mathcal{S}_{6}\right)$ for $\mathcal{A}=\mathcal{Y}, \mathcal{L}$, or $\mathcal{R}$ and the fact that all variables square to 1 , it can be shown that the right-hand side of Eq. (10a) is equal to the product of the right-hand sides of the other four equations. Thus we have the contradiction $1=-1$, showing that no distance- $d$ communication-assisted LHV model reproduces the predictions of quantum mechanics in this instance.

For other values of $n \neq 12 f$, with $f$ odd, an identical contradiction applies to a graph consisting of $r=(n$ -12 ) mod 24 unconnected nodes and a ring of size $n-r$. It is also possible to adapt our example to two-dimensional cluster states. One can show, for example, that for a $(3 f+3)$ $\times(3 f+3)$ cluster state, with $f$ odd, a communication distance of at least $2 f$ is required.

\section{B. Site-invariant communication protocols}

Both the numbering and the arrangement of nodes in a graph are arbitrary, so it seems reasonable to suppose that a communication protocol should be insensitive to these things. We refer to this property as site invariance and define it formally as follows. Given a graph $\mathcal{G}$, each of whose nodes has been assigned a measurement, a permutation that leaves the graph invariant is one that interchanges nodes and their measurements, letting edges move with the nodes, such that the new graph $\mathcal{G}^{\prime}$ is identical to $\mathcal{G}$ in the sense that they could be placed on top of each other with all nodes, measurements, and edges overlapping. A site-invariant protocol is one for which nodes in identical situations, as defined by permutations that leave the graph invariant, make the same signflipping decision. Surprisingly, we find this trait to be at odds with the modeling of submeasurements. 


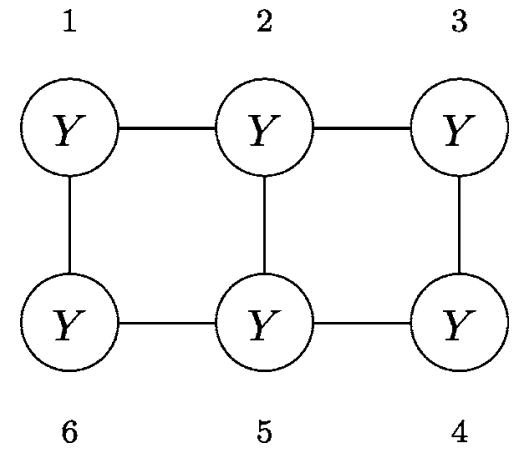

FIG. 2. Example demonstrating that any communicationassisted LHV model based on the hidden variables of Eq. (2) and assisted by a site-invariant communication protocol fails to reproduce some submeasurements. The global measurement $M$ $=Y_{1} Y_{2} Y_{3} Y_{4} Y_{5} Y_{6}$ has a random outcome, but contains a submeasurement $\tilde{M}=Y_{1} Y_{2} Y_{3} I_{4} Y_{5} I_{6}$ such that $-\tilde{M}$ is an element of the stabilizer group. This means that an overall sign flip is required to correct the +1 prediction of the hidden variables for a measurement of $\tilde{M}$. The two qubits measuring $Y$ at nodes 1 and 3 are in symmetric situations, as are the qubits at nodes 2 and 5. Thus, under a site-invariant protocol, 1 and 3 must make the same sign-flipping decision, as must 2 and 5. For each pair, the sign-flipping decisions cancel one another, producing no overall sign flip and thus giving an incorrect result of +1 for the measurement of $\tilde{M}$.

We demonstrate the limitations imposed by site invariance using the example of a $2 \times 3$ cluster state, which is depicted in Fig. 2. The two relevant measurements for this example are $M=Y_{1} Y_{2} Y_{3} Y_{4} Y_{5} Y_{6}$, which has a random outcome, and $\tilde{M}=Y_{1} Y_{2} Y_{3} I_{4} Y_{5} I_{6}$, which has the certain outcome -1 . When either of these is considered as a global measurement, our model yields the correct prediction, as we have already shown in general, but when the second is considered as a submeasurement of the first, the model fails. In this second case, rules (1)-(4) say that the two qubits measuring $Y$ at nodes 2 and 5 should introduce a sign flip, but the two qubits measuring $Y$ at nodes 1 and 3 should not. The result is no overall sign flip and an outcome +1 , showing that the model gets the submeasurement outcome wrong. In contrast, when $\widetilde{M}$ is considered as a global measurement, rules (1)-(4) dictate a sign flip for qubit 2, but no other qubit, thus giving the correct, certain outcome -1 . The same measurement $\tilde{M}$ can lead to different sign-flipping decisions in the two situations because the nearest-neighbor environments of the qubits differ depending on whether a submeasurement or a global measurement is under consideration. As is shown in Fig. 2, the counterexample is not limited to the communication model used in this paper. In fact, any site-invariant protocol based on our hidden variables yields an incorrect result for the submeasurement $\tilde{M}$.

This example can easily be generalized by adding $p$ rows and $q$ columns to opposite sides of the $2 \times 3$ cluster state. Doing this results in a class of $(2+2 p) \times(3+2 q)$ cluster states for which LHV models based on the hidden variables of Eq. (2) and assisted by a site-invariant communication protocol fail for some submeasurements.

\section{Site-invariant model for $1 D$ cluster states}

In Sec. IV A it was shown that there exist graph states of size $n$ for which any communication-assisted LHV model must involve communication over a distance at least $n / 6$ if it reproduces all subcorrelations. Note that this result applies to all models, whether site-invariant or not, and whatever the structure of the LHVs. In Sec. IV B it was shown that, for certain graph states, no model based on the hidden variables of Eq. (2) and assisted by a site-invariant communication protocol is capable of reproducing all subcorrelations. This result holds even if the model allows unlimited-distance communication.

It is intriguing that both of these results apply to two-dimensional cluster states, since two-dimensional cluster states, along with single-qubit measurements, are universal for quantum computation. ${ }^{1}$ It is therefore reasonable to ask whether the same results hold for onedimensional cluster states (linear chains), which are not universal for computation [13]. In this subsection we show that linear chains do permit successful site-invariant protocols. The protocol we describe involves communication over a distance equal to the number of edges in the one-dimensional cluster state (i.e, unlimited communication). At present it is unknown whether the subcorrelations of a linear chain could be reproduced by a protocol with limited-distance communication.

The fact that unlimited communication is allowed is in the same spirit as our counterexample of Fig. 2, where communication spans the entire graph and the only restriction is site invariance. The key simplification in the case of onedimensional cluster states is that all qubits, except those at the ends of the chain, have exactly two neighbors. As a consequence, the form of stabilizer elements whose hiddenvariable result from Eq. (2) requires correction is constrained so that the correction can be effected by a site-invariant protocol.

For an $n$-qubit chain, the $n$ stabilizer generators are given by $G_{1}=X_{1} Z_{2}, G_{j}=Z_{j-1} X_{j} Z_{j+1}$ for $j=2, \ldots, n-1$, and $G_{n}$ $=Z_{n-1} X_{n}$. Any stabilizer element is a product of generators. An arbitrary product of generators can be decomposed into a product of terms each of which is a product of successive generators. We call these terms primitive stabilizers or just primitives. The primitive stabilizers are separated by the omission of one or more generators in the product of generators. An example of a stabilizer element for $n=10$ qubits is $G_{1} G_{2} G_{3} G_{5} G_{6} G_{9}=-Y_{1} X_{2} Y_{3} I_{4} Y_{5} Y_{6} Z_{7} Z_{8} X_{9} Z_{10}$. The primitives in this example are $G_{1} G_{2} G_{3}, G_{5} G_{6}$, and $G_{9}$.

Associated with each primitive is a Pauli product (with the sign omitted) for the qubits corresponding to the generators in the primitive. We call these Pauli products words. For the 10-qubit example above, the words are $Y_{1} X_{2} Y_{3}, Y_{5} Y_{6}$, and $X_{9}$. At each end of a word, there is an $I$ if one generator is omitted and a $Z$ if two or more generators are omitted. We can make these word boundaries apply even at the end of the

\footnotetext{
${ }^{1}$ Admittedly, our models are only concerned with measurements of Pauli operators, which are not universal for computation due to the Gottesman-Knill theorem [12].
} 
linear chain by embedding our cluster state in an infinite linear chain. The generators for the qubits to the left of $j$ $=1$ and to the right of $j=n$ are always omitted, and we redefine $G_{1}=Z_{0} X_{1} Z_{2}$ and $G_{n}=Z_{n-1} X_{n} Z_{n+1}$.

If a word is bounded by an $I$, there must be another word immediately on the other side of the I. A sentence is a Pauli product consisting of a set of words separated by singleton Is and bracketed by $Z s$ at both ends. Words are not stabilizer elements, but sentences are. The example above contains two sentences, $Z_{0} Y_{1} X_{2} Y_{3} I_{4} Y_{5} Y_{6} Z_{7}$ (including the zeroth qubit) and $Z_{8} X_{9} Z_{10}$. The $Z$ bookends on a sentence separate it from other, nonoverlapping sentences in the same overall stabilizer element. Between the Zs in successive sentences, there can be an arbitrary number of Is. Any stabilizer element is a product of nonoverlapping sentences.

We can list the entire set of words by considering all possible primitives:

$X$ for a primitive with one Pauli operator,

$Y \otimes Y$ for a primitive with two Pauli operators,

$Y \otimes X^{\otimes(j-2)} \otimes Y$ for a primitive with $j \geq 3$ Pauli operators.

For stabilizer elements, Is occur only between sentences or as singletons between words, $X \mathrm{~s}$ and $Y$ s occur only in words, and $Z s$ occur only as the boundaries of sentences.

Recall that the goal of the communication protocol is to introduce a sign flip into the product of hidden-variable entries for those Pauli products that are the negative of a stabilizer element. The only words that introduce a minus sign into the corresponding product of generators are those of the form $Y \otimes X^{\otimes(j-2)} \otimes Y$ with $j$ odd. Thus a candidate for a siteinvariant communication protocol is the following.

(1) Each site at which an $X$ or a $Z$ is measured broadcasts the measurement performed upon it.

(2) Each site that measures $X$ determines if it is the middle (implying an odd number of $X \mathrm{~s}$ ) qubit in a word of the form (11c) in a submeasurement sentence, and if so, flips its hidden-variable entry, i.e., changes $x$ to $-x$.

This clearly gets any stabilizer right and thus all global correlations right.

The only question remaining is whether this protocol works for subcorrelations. We answer this question by showing the following: two sentences, $S_{1}$ and $S_{2}$, that are submeasurements of the same global measurement, generally not a stabilizer element, must be identical on the region where they overlap, except possibly at bracketing Zs. This property implies that $S_{1}$ and $S_{2}$ have exactly the same words in the region of overlap. Thus, for any pair of submeasurements of the global measurement, a sign flip arising from a word of the form (11c) in the overlap region is common to both submeasurements. Since both the word and the sign flip occur in both submeasurements, our protocol correctly predicts both outcomes.
To prove this property, notice first that if $S_{1}$ and $S_{2}$ overlap (should they not overlap, the property is trivially true), there are two cases: The region of overlap coincides with one of the sentences, or it does not. In the former case, we choose $S_{2}$ to be the sentence that coincides with the region of overlap, and in the latter case, we choose $S_{1}$ to be the sentence on the left and $S_{2}$ to be the one on the right. With these conventions, the left boundary of the overlap region coincides with the $Z$ that bounds the left end of $S_{2}$, and the right boundary of the overlap region coincides in the former (latter) case with the $Z$ that bounds the right end of $S_{2}\left(S_{1}\right)$.

To be submeasurements of the same global measurement, the two sentences must satisfy the following basic rule: In the overlap region, sites within a word of one sentence must be occupied in the other sentence by the same Pauli operator or by an $I$. Since Zs do not occur in words, this rule implies that the $Z$ s that bound the overlap region at either end in one of the two sentences cannot occupy a site within a word in the other sentence and thus must be a bounding $Z$ or a singleton $I$ in the other sentence. The submeasurement requirement, by itself, implies that in the overlap region, the site of a singleton $I$ in one sentence can be occupied by anything in the other sentence, but the available words impose a much stronger constraint, as we now show.

Consider the left boundary of the overlap region, which is occupied by the leftmost $Z$ in $S_{2}$ and by a $Z$ or a singleton $I$ in $S_{1}$. Immediately to the right in both $S_{1}$ and $S_{2}$ is a word. When one of these words is shorter than the other, the basic rule implies that the shorter word must be a prefix of the longer one. A glance at the allowed words in Eq. (11) shows, however, that none is a prefix of another. Thus $S_{1}$ and $S_{2}$ must have the same word in this first overlap position, which is followed by a singleton $I$ in both sentences. Applying the same logic to this and subsequent singleton Is shows, as promised, that $S_{1}$ and $S_{2}$ are identical in the overlap region, except possibly at the boundaries.

\section{CONCLUSION}

Communication-assisted LHV models allow us to explore the degree of nonlocality present in various states. In this paper we focused on graph states and parameterized communication-assisted LHV models by the allowed distance of communication, where the distance between two qubits is defined as the number of links between the corresponding nodes in its graph. Interestingly, a simple nearestneighbor communication protocol is capable of yielding the global quantum-mechanical correlation for any measurement of Pauli products on any graph state, but the submeasurements of these global measurements are much harder to reproduce. To replicate the predictions of quantum mechanics for all submeasurements on any graph state, it is necessary for the communication distance to scale as $n / 6$ or faster in the number $n$ of qubits in the graph. Thus, using the metric of communication distance, reproduction of all subcorrelations is a much more difficult task than producing global correlations.

Unexpectedly, another property of interest for communication protocols seems to be a kind of graph isomorphism 
symmetry, which we dubbed site invariance. By considering a class of two-dimensional cluster states, we showed that, regardless of communication distance, site-invariant communication protocols based on the local hidden variables of Eq. (2) are incapable of yielding the correct correlations for all submeasurements on all graph states. Nevertheless, a siteinvariant communication protocol with unlimited communication distance is capable of yielding the correct correlations for all submeasurements on all one-dimensional cluster states. These results are notable because the two-dimensional cluster state is a suitable resource for measurement-based quantum computation, while the one-dimensional cluster state is not. This perhaps suggests a fundamental division between states such as the two-dimensional cluster state which are sufficient for quantum computation and states such as the GHZ and one-dimensional cluster state which are not.
Our hope is that the study of communication-assisted LHV models will lead to a better understanding of the nature of entanglement and the apparent nonlocality of quantum mechanics. Already, in this paper, we have indications that the richness of entanglement lies not in the overall measurement result, but in measurement subcorrelations.

\section{ACKNOWLEDGMENTS}

Research at the Perimeter Institute was supported in part by the Government of Canada through NSERC and by the Province of Ontario through MEDT. C. M. C., B. E., and M. B. E. are partly supported by Army Research Office Contract No. W911NF-04-1-0242. S. P. acknowledges support by the Belgian American Educational Foundation, by the National Science Foundation under Grant No. EIA-0086038, and by the EU project QIP.
[1] D. Schlingemann, e-print quant-ph/0111080.

[2] M. Van den Nest, J. Dehaene, and B. De Moor, Phys. Rev. A 69, 022316 (2004).

[3] H. J. Briegel and R. Raussendorf, Phys. Rev. Lett. 86, 910 (2001).

[4] R. Raussendorf and H. J. Briegel, Phys. Rev. Lett. 86, 5188 (2001).

[5] M. Hein, W. Dür, J. Eisert, R. Raussendorf, M. Van den Nest, and H.-J. Briegel, e-print quant-ph/0602096.

[6] O. Gühne, G. Tóth, P. Hyllus, and H. J. Briegel, Phys. Rev. Lett. 95, 120405 (2005).

[7] V. Scarani, A. Acín, E. Schenck, and M. Aspelmeyer, Phys.
Rev. A 71, 042325 (2005)

[8] T. E. Tessier, C. M. Caves, I. H. Deutsch, B. Eastin, and D. Bacon, Phys. Rev. A 72, 032305 (2005).

[9] M. A. Nielsen and I. L. Chuang, Quantum Computation and Quantum Information (Cambridge University Press, Cambridge, England, 2000), Sec. 10.5.3.

[10] R. Diestel, Graph Theory (Springer-Verlag, New York, 2000).

[11] M. B. Elliott, B. Eastin, and C. M. Caves (in preparation).

[12] S. Anders and H. J. Briegel, Phys. Rev. A 73, 022334 (2006).

[13] M. Van den Nest, A. Miyake, W. Dür, and H. J. Briegel, Phys. Rev. Lett. 97, 150504 (2006). 